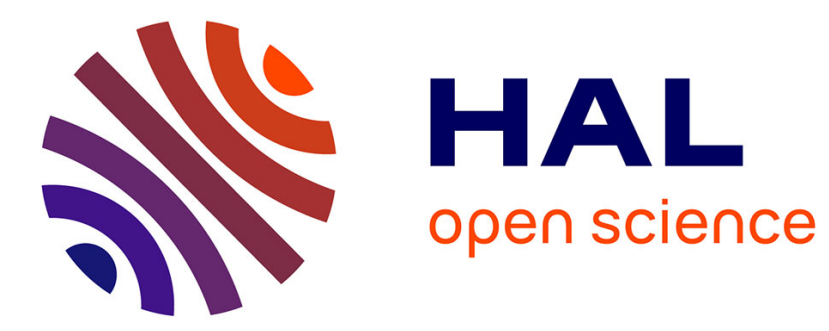

\title{
Blackbody Theory for Hyperbolic Materials
}

Svend-Age Biehs, Slawa Lang, Alexander Petrov, Manfred Eich, Philippe Ben-Abdallah

\section{To cite this version:}

Svend-Age Biehs, Slawa Lang, Alexander Petrov, Manfred Eich, Philippe Ben-Abdallah. Blackbody Theory for Hyperbolic Materials. Physical Review Letters, 2015, 115 (17), pp.174301. 10.1103/PhysRevLett.115.174301 . hal-01335142

\section{HAL Id: hal-01335142 \\ https://hal-iogs.archives-ouvertes.fr/hal-01335142}

Submitted on 21 Jun 2016

HAL is a multi-disciplinary open access archive for the deposit and dissemination of scientific research documents, whether they are published or not. The documents may come from teaching and research institutions in France or abroad, or from public or private research centers.
L'archive ouverte pluridisciplinaire HAL, est destinée au dépôt et à la diffusion de documents scientifiques de niveau recherche, publiés ou non, émanant des établissements d'enseignement et de recherche français ou étrangers, des laboratoires publics ou privés. 


\title{
Blackbody Theory for Hyperbolic Materials
}

\author{
Svend-Age Biehs, ${ }^{1, *}$ Slawa Lang, ${ }^{2}$ Alexander Yu. Petrov, ${ }^{2,3}$ Manfred Eich, ${ }^{2}$ and Philippe Ben-Abdallah ${ }^{4,}$ \\ ${ }^{1}$ Institut für Physik, Carl von Ossietzky Universität, D-26111 Oldenburg, Germany \\ ${ }^{2}$ Institute of Optical and Electronic Materials, Hamburg University of Technology, 21073 Hamburg, Germany \\ ${ }^{3}$ ITMO University, 49 Kronverskii Ave., 197101 St. Petersburg, Russia \\ ${ }^{4}$ Laboratoire Charles Fabry, UMR 8501, Institut d'Optique, CNRS, Université Paris-Sud 11, \\ 2, Avenue Augustin Fresnel, 91127 Palaiseau Cedex, France \\ (Received 15 February 2015; published 22 October 2015)
}

\begin{abstract}
The blackbody theory is revisited in the case of thermal electromagnetic fields inside uniaxial anisotropic media in thermal equilibrium with a heat bath. When these media are hyperbolic, we show that the spectral energy density of these fields radically differs from that predicted by Planck's blackbody theory and that the maximum of the spectral energy density determined by Wien's law is redshifted. Finally, we derive the Stefan-Boltzmann law for hyperbolic media which becomes a quadratic function of the heat bath temperature.
\end{abstract}

DOI: 10.1103/PhysRevLett.115.174301

In 1901, Planck [1] derived the famous law describing the spectral distribution of energy of a blackbody (BB) by introducing the concept of quantum of light, so laying the foundation of quantum physics. In his description of the problem, the electromagnetic field inside a cavity made with opaque walls, which is set at a constant temperature, is studied. In this formulation [2], the cavity is at thermal equilibrium and acts as a heat bath. The walls of the cavity emit and absorb electromagnetic waves so that the field itself becomes equilibrated. The internal energy density of the electromagnetic field in the cavity with volume $V$ for both principal polarization states (abbreviated by $s$ and $p$ ) is then given by

$U_{\mathrm{BB}}^{s / p}=\frac{1}{2} \int_{0}^{\infty} d \omega \frac{\omega^{2}}{\pi^{2} c^{3}} \frac{\hbar \omega}{e^{\hbar \omega / k_{B} T}-1}=\frac{1}{2} \frac{\pi^{2}}{15} \frac{\left(k_{B} T\right)^{4}}{(\hbar c)^{3}}$,

where $\hbar, k_{B}$, and $c$ denote Planck's constant, Boltzmann's constant, and the velocity of light in vacuum. Here and in the following we neglect vacuum fluctuations.

In this work, we revisit this old problem when the cavity is filled with a uniaxial medium [3] with a relative permittivity tensor of the form $\boldsymbol{\epsilon}=\operatorname{diag}\left(\epsilon_{\perp}, \epsilon_{\perp}, \epsilon_{\|}\right)$. Here, without loss of generality, we assume that the optical axis points into the $z$ direction; $\epsilon_{\|}$is the permittivity along the optical axis and $\epsilon_{\perp}$ is the permittivity perpendicular to the optical axis. Since we are interested in ideal black body laws (the Planck, Wien, and Stefan-Boltzmann laws), we neglect dispersion, dissipation, and nonlocal effects in the following if not specified differently. Within such materials so-called ordinary modes (OMs) and extraordinary modes (EMs) exist and satisfy the dispersion relations [3]

$$
\frac{k_{\perp}^{2}}{\epsilon_{\perp}}+\frac{k_{\|}^{2}}{\epsilon_{\perp}}=\frac{\omega^{2}}{c^{2}}
$$

PACS numbers: 44.05.+e, 12.20.-m, 44.40.+a, 78.67.-n

$$
\frac{k_{\perp}^{2}}{\epsilon_{\|}}+\frac{k_{\|}^{2}}{\epsilon_{\perp}}=\frac{\omega^{2}}{c^{2}},
$$

where $k_{\perp}\left(k_{\|}\right)$is the wave number component perpendicular (parallel) to the optical axis. In usual dielectric uniaxial media the principal constants $\epsilon_{\perp}$ and $\epsilon_{\|}$are both positive and the isofrequency surfaces defined by relations (2) and (3) are spheres or ellipsoids, respectively, as illustrated in Fig. 1(a). On the other hand, when $\epsilon_{\|}<0$ and $\epsilon_{\perp}>0$ or $\epsilon_{\|}>0$ and $\epsilon_{\perp}<0$ the isofrequency surfaces of the EM are two- or onesheeted hyperboloids [see Fig. 1(b)]. The first class of such a uniaxial medium is called hyperbolic medium (HM) of type I while the second one HM of type II [4,5]. Of course both $\epsilon_{\perp}$ and $\epsilon_{\|}$can also be negative. In such uniaxial metalliclike materials no propagating modes exist and the upcoming quantities are all 0 .
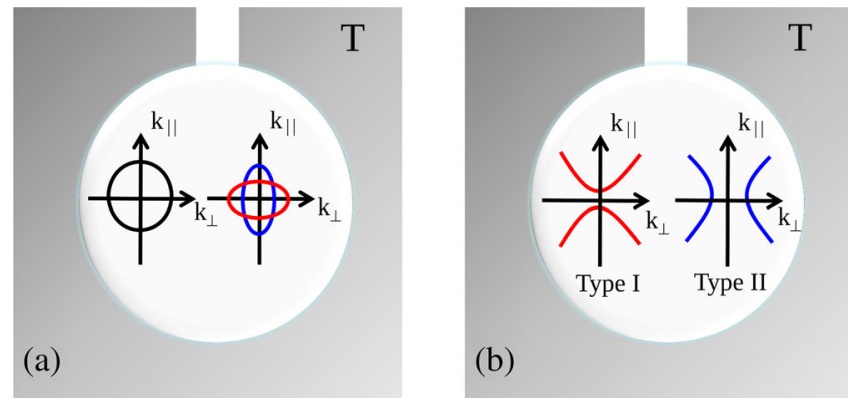

FIG. 1 (color online). (a) Cavity at temperature $T$ containing an isotropic medium of permittivity $\epsilon>0$ or an anisotropic (uniaxial) medium with $\epsilon_{\perp}>0$ and $\epsilon_{\|}>0$. The particular case $\epsilon_{\perp}=$ $\epsilon_{\|}=1$ corresponds to the classical BB. (b) Cavity at temperature $T$ containing a HM of type I $\left(\epsilon_{\perp}>0\right.$ and $\left.\epsilon_{\|}<0\right)$ and of type II $\left(\epsilon_{\perp}<0\right.$ and $\left.\epsilon_{\|}>0\right)$. The isofrequency curves are plotted inside the cavities in the plane $\left(k_{\perp}, k_{\|}\right)$. 
To start, we focus our attention on the electromagnetic field inside a cavity filled with an arbitrary uniaxial medium. The spectral density of states (DOS) defined as the energy density $U$ normalized to the mean energy of a harmonic oscillator and associated to the thermal field can be calculated either by counting the modes in the wave vector space using expressions (2) and (3) or by means of the generalized trace formula [6-8]

$$
D(\omega)=\frac{\omega}{c^{2} \pi} \operatorname{Im} \operatorname{Tr}\left[\mathbb{G}^{\mathrm{EE}}(\mathbf{r}, \mathbf{r}, \omega)+\boldsymbol{\mu} \mathbb{G}^{\mathrm{HH}}(\mathbf{r}, \mathbf{r}, \omega)\right],
$$

which can be derived by using the fluctuation dissipation theorem (FDT) $[16,17]$ or by an eigenmode analysis as in Ref. [7] without using the FDT. Here $\mathbb{G}^{\mathrm{EE}}$ and $\mathbb{G}^{\mathrm{HH}}$ are the electric and magnetic Green's dyadics for the bulk material and $\boldsymbol{\mu}$ is the relative permeability tensor. The result for a classical BB can be retrieved by using $\boldsymbol{\epsilon}=\boldsymbol{\mu}=\mathbb{1}$ with the unit dyad 1 . Then the above expression reduces to the wellknown expression $D_{\mathrm{BB}}^{s+p}(\omega)=\omega^{2} / \pi^{2} c^{3}$. In the following, for the sake of clarity, we consider nonmagnetic materials (i.e., $\boldsymbol{\mu}=\mathbb{1}$ ) only. By inserting the general expression of dyadic Green's tensors of uniaxial materials $[18,19]$ in the trace formula (4) it is straightforward to derive the DOS for the three different classes of uniaxial media. Assuming that those media are lossless then in dielectric anisotropic media the DOS $D_{D}^{o}$ for the OMs and $D_{D}^{e}$ for the EMs are given by the expressions (see the Supplemental Material [8])

$$
D_{D}^{o}(\omega)=\frac{\omega^{2}}{\pi^{2} c^{3}} \frac{\epsilon_{\perp} \sqrt{\epsilon_{\perp}}}{2} \quad \text { and } \quad D_{D}^{e}(\omega)=\frac{\omega^{2}}{\pi^{2} c^{3}} \frac{\epsilon_{\|} \sqrt{\epsilon_{\perp}}}{2},
$$

which where already derived by Eckhardt [20], for instance. On the other hand, in the hyperbolic case we obtain (see the Supplemental Material [8])

$$
\begin{gathered}
D_{\mathrm{I}}^{o}=\frac{\omega^{2}}{\pi^{2} c^{3}} \frac{\epsilon_{\perp} \sqrt{\epsilon_{\perp}}}{2} \\
D_{\mathrm{I}}^{e}=\frac{\omega}{\pi^{2} c^{2}} \frac{\left|\epsilon_{\|}\right|}{2}\left(\sqrt{k_{\perp, \max }^{2} \frac{\epsilon_{\perp}}{\left|\epsilon_{\|}\right|}+\frac{\omega^{2}}{c^{2}} \epsilon_{\perp}}-\frac{\omega}{c} \sqrt{\epsilon_{\perp}}\right)
\end{gathered}
$$

and

$$
\begin{gathered}
D_{\mathrm{II}}^{o}=0, \\
D_{\mathrm{II}}^{e}=\frac{\omega}{\pi^{2} c^{2}} \frac{\epsilon_{\|}}{2} \sqrt{k_{\perp, \max }^{2} \frac{\left|\epsilon_{\perp}\right|}{\epsilon_{\|}}-\frac{\omega^{2}}{c^{2}}\left|\epsilon_{\perp}\right|},
\end{gathered}
$$

for the type I and type II media, respectively. We have checked that the method of counting modes in $k$ space gives the same results. This is another way to obtain the results for the LDOS without using the FDT. Note that we have introduced a cutoff wave number $k_{\perp, \max }$, which for dispersive media can be a function of the frequency and which is determined by the real (atomic or meta) structure of the medium. For an ideal $\mathrm{HM} k_{\perp \text {,max }}$ is infinity so that the DOS diverges as was pointed out previously [21,22]. However, for any real structure $k_{\perp \text {,max }}$ is a finite quantity [23]. For hyperbolic metamaterials (HMMs) it is mainly determined by the unit-cell size of the metastructure. Note further that the DOS of the EMs of type I and type II HMs coincides for $k_{\perp, \max } \gg \frac{\omega}{c} \sqrt{\left|\epsilon_{\|}\right|}$and is given by

$$
D_{\mathrm{I}}^{e} \approx D_{\mathrm{II}}^{e} \approx \frac{\omega}{\pi^{2} c^{2}} \frac{\sqrt{\left|\epsilon_{\perp} \epsilon_{\|}\right|}}{2} k_{\perp, \max } .
$$

Apparently the DOS is linear in $\omega$ and linear in $k_{\perp, \text { max }}$. If $k_{\perp, \max }$ would be proportional to $\omega / c$ we would retrieve the usual quadratic behavior of the DOS with respect to frequency. We want to emphasize that the approximate expression of the DOS for a nonideal dispersive material given in Refs. [21,22] is proportional to $k_{\perp, \max }^{3}$.

With the help of the DOS we can determine the thermodynamic potentials of the photon gas inside the uniaxial material. By definition, the internal and the free energy per unit volume are given by [17]

$$
\left\{\begin{array}{l}
U \\
F
\end{array}\right\}=\int_{0}^{\infty} d \omega D(\omega)\left\{\begin{array}{l}
\mathcal{U}(\omega, T) \\
\mathcal{F}(\omega, T)
\end{array}\right\},
$$

where

$$
\begin{gathered}
\mathcal{U}(\omega, T)=\frac{\hbar \omega}{\mathrm{e}^{\hbar \omega / k_{B} T}-1}, \\
\mathcal{F}(\omega, T)=k_{B} T \ln \left[1-e^{-\left(\hbar \omega / k_{B} T\right)}\right] .
\end{gathered}
$$

Finally, from the internal and free energy we can also determine the entropy per unit volume by $S=(U-F) / T$. Clearly, by means of these expressions we can derive any thermodynamic property of the photon gas inside the cavity as the pressure $P=U / 3$, the photonic heat capacity $C_{V}=\partial U / \partial T$, etc.

Let us first have a look at the expressions for the cavity filled with an ordinary uniaxial material. In this case, we obtain

$$
U_{D}^{o}=U_{\mathrm{BB}}^{s} \epsilon_{\perp} \sqrt{\epsilon_{\perp}} \quad \text { and } \quad U_{\mathrm{D}}^{e}=U_{\mathrm{BB}}^{p} \epsilon_{\|} \sqrt{\epsilon_{\perp}} .
$$

Therefore, when $\epsilon_{\perp}=\epsilon_{\|}=1$ we recover the classical BB result. The relations between the internal energy, the free energy, and the entropy have the familiar forms

$$
F_{D}^{o / e}=-\frac{1}{3} U_{D}^{o / e} \quad \text { and } \quad S_{D}^{o / e}=\frac{4}{3} \frac{U_{D}^{o / e}}{T}
$$

Note that these relations are the same as for a usual BB because the DOS of the field inside a dielectric uniaxial medium is proportional to $\omega^{2}$. 
On the contrary, in type I and type II HMs we have seen that the DOS of the EMs is linear in $\omega$ as in a 2D photon gas in vacuum. It follows that the relations between the thermodynamic properties of the photon gas are radically different in that case. Indeed, we obtain $\left(k_{\perp, \max } \gg \sqrt{\left|\epsilon_{\|}\right|} \omega / c\right)$

$$
U_{\mathrm{I} / \mathrm{II}}^{e} \approx \frac{\sqrt{\left|\epsilon_{\|} \epsilon_{\perp}\right|}}{2} k_{\perp, \max } \frac{2}{\pi^{2} c^{2}} \frac{\zeta(3)}{\hbar^{2}}\left(k_{B} T\right)^{3},
$$

where $\zeta$ is the zeta function. Furthermore, we find

$$
F_{\mathrm{I} / \mathrm{II}}^{e}=-\frac{1}{2} U_{\mathrm{I} / \mathrm{II}}^{e} \quad \text { and } \quad S_{\mathrm{I} / \mathrm{II}}^{e}=\frac{3}{2} \frac{U_{\mathrm{I} / \mathrm{II}}^{e}}{T} .
$$

Hence $U, F$, and $S$ are proportional to $T^{3}$ and not anymore to $T^{4}$. Naturally, for the OMs we find

$$
U_{\mathrm{I}}^{o}=U_{D}^{o} \quad \text { and } \quad U_{\mathrm{II}}^{o}=0 .
$$

Note that for the type II HMs the internal energy of the OMs is zero, since there are no OMs in such a material. The internal energy of the OMs in a type I HM is just the same as in a dielectric uniaxial medium. Hence, the relations between the thermodynamic potentials are the same as in a dielectric uniaxial medium. However, in typical HMs the maximal wave vector is much larger than the vacuum wave vector $k_{\perp, \text { max }} \gg \omega / c$ making the material properties dominated by the EMs.

Another consequence of the linearity of the DOS with respect to $\omega$ inside a HM is the spectral shift of Wien's frequency $\omega_{\max }$ (wavelength $\lambda_{\max }$ ) at which the energy distribution function has its maximum. For both type I and type II HMs we find after a straightforward calculation from relations (9) and (11) $\left(k_{\perp, \text { max }} \gg \sqrt{\left|\epsilon_{\|}\right|} \omega / c\right)$ that this maximum is reached when

$$
\frac{\hbar \omega_{\max }}{k_{B} T}=1.59 \quad \text { or } \quad \frac{2 \pi l_{c}}{\lambda_{\max }}=3.92,
$$

whereas for a usual BB $\hbar \omega_{\max } / k_{B} T=2.82$ and $2 \pi l_{c} / \lambda_{\max }=4.965$. Here we have introduced the thermal coherence length $l_{c} \equiv \hbar c / k_{B} T$ [6]. Hence, we see that Wien's frequency is shifted toward smaller values and the maximum vacuum wavelength to larger values (see Fig. 2).

It is now interesting to compare the internal energy of the EMs in a HM with that of a classical BB. From expressions (1) and (16) we immediately get

$$
\frac{U_{\mathrm{I} / \mathrm{II}}^{e}}{U_{\mathrm{BB}}^{p}} \approx \sqrt{\left|\epsilon_{\|} \epsilon_{\perp}\right|}\left(k_{\perp, \max } l_{c}\right) \frac{30}{\pi^{4}} \zeta(3) .
$$

If $\Lambda$ denotes the unit-cell size of our HM then $k_{\|, \max }=\pi / \Lambda$ or $k_{\perp, \max }=\pi / \Lambda$ depending on the concrete structure. For example, for a multilayer structure with layers perpendicular to the $z$ axis the edge of the first Brillouin

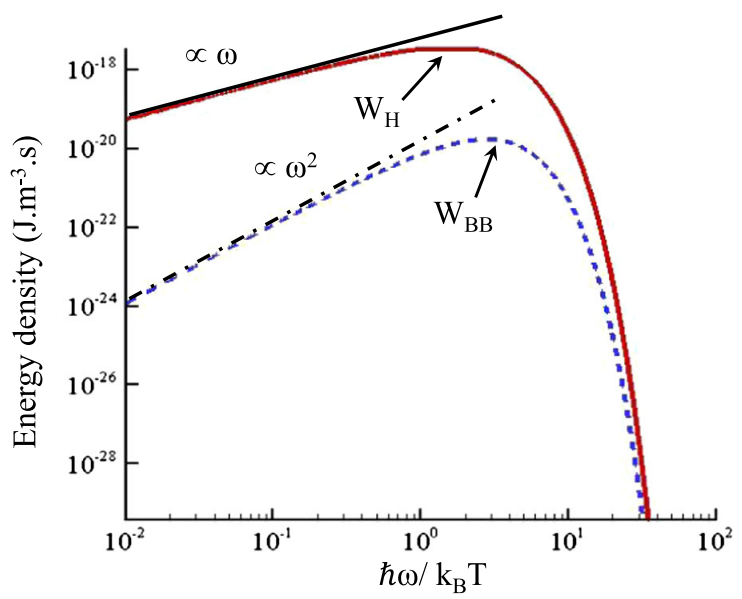

FIG. 2 (color online). Spectral energy density (in log-log scale) of a type I and type II hyperbolic BB (red solid line) with $\left|\epsilon_{\|} \epsilon_{\perp}\right|=1$ and $k_{\perp, \max }=\pi / \Lambda$ with $\Lambda=50 \mathrm{~nm}$ at $T=300 \mathrm{~K}$. This distribution is compared with the classical BB spectrum (blue dashed line). The solid and dashed straight lines show the asymptotic behavior in $\omega$ and $\omega^{2}$ of the hyperbolic and classical BB spectrum. The arrows indicate Wien's frequencies in both cases.

zone gives $k_{\|, \max }=\pi / \Lambda$, whereas for a nanowire structure with nanowires along the $z$ axis the edge of the first Brillouin zone gives $k_{\perp, \max }=\pi / \Lambda$. However, independent of the structure both maximum wave vectors are related by $k_{\perp, \max } \approx \sqrt{\left|\epsilon_{\|} / \epsilon_{\perp}\right|} k_{\|, \text {max }}$ [see Eq. (3)] so that we generally have $U_{\mathrm{I} / \mathrm{II}}^{e} / U_{\mathrm{BB}}^{p} \propto l_{c} / \Lambda$. At a temperature of $300 \mathrm{~K}$ the coherence length is $l_{c}=7.6 \mu \mathrm{m}$. The period of realistic HMMs is typically larger than $\Lambda \approx 10 \mathrm{~nm}$ (see Ref. [24] for instance). In natural HMs [23,25-27] there might be different length scales which determine their hyperbolic behavior. Nonetheless, we think that it is reasonable to assume that the main length scale is again given by the unit cell size which is in this case determined by the interatomic spacing, i.e., $\Lambda \approx 1 \AA$. Hence, the internal energy of thermal radiation inside a $\mathrm{HM}$ can be $3(\Lambda \approx 10 \mathrm{~nm})$ to $5(\Lambda \approx 1 \AA)$ orders of magnitude larger than that of a perfect BB. The same is of course also true for the free energy and the entropy. This result suggests that the radiative heat flux inside a HM is dramatically enhanced compared to that of a classical BB.

In order to evaluate the flux radiated by a cavity filled with a HM into a HM and to derive the Stefan-Boltzmann law, we calculate now the Poynting vector outside the cavity in the direction of the principal optical axis by assuming, for convenience, that the cavity opening (see Fig. 3) is along this axis. To this end, we rely on Rytov's fluctuational electrodynamics. Within this theoretical framework the thermal fluctuations on a microscopical scale result in macroscopic fluctuating source currents $\mathbf{j}$ which are the sources of fluctuational thermal electromagnetic fields determined by 


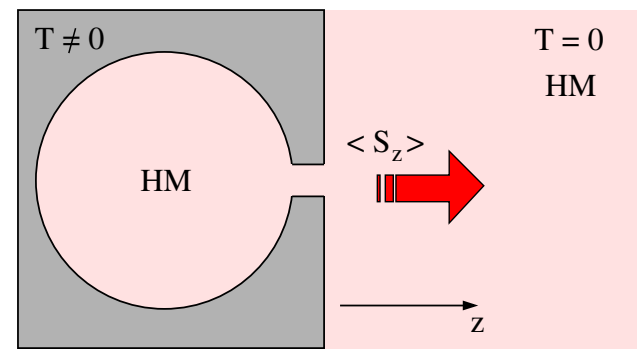

FIG. 3 (color online). Sketch of a hyperbolic BB emitter. The $\mathrm{HM}$ inside and outside the cavity are the same.

$$
\begin{aligned}
& \mathbf{E}(\mathbf{r} ; \omega)=i \omega \mu_{0} \int_{V} d^{3} r^{\prime} \mathbb{G}^{\mathrm{EE}}\left(\mathbf{r}, \mathbf{r}^{\prime} ; \omega\right) \mathbf{j}\left(\mathbf{r}^{\prime} ; \omega\right), \\
& \mathbf{H}(\mathbf{r} ; \omega)=i \omega \mu_{0} \int_{V} d^{3} r^{\prime} \mathbb{G}^{\mathrm{HE}}\left(\mathbf{r}, \mathbf{r}^{\prime} ; \omega\right) \mathbf{j}\left(\mathbf{r}^{\prime} ; \omega\right) .
\end{aligned}
$$

Here, $V$ is the volume of the considered structure containing the source currents which is in our case the filled cavity; $\mu_{0}$ is the permeability of vacuum. Note that $\mathbb{G}^{\mathrm{HE}}\left(\mathbf{r}, \mathbf{r}^{\prime}\right)=$ $\left(1 / i \omega \mu_{0}\right) \nabla \times \mathbb{G}^{\mathrm{EE}}\left(\mathbf{r}, \mathbf{r}^{\prime}\right)$. If the filled cavity is held in local thermal equilibrium at a given temperature $T$, the source currents fulfill the FDT of the second kind [28],

$$
\begin{aligned}
\left\langle j_{\alpha}(\mathbf{r}, \omega) j_{\beta}^{*}\left(\mathbf{r}^{\prime}, \omega^{\prime}\right)\right\rangle= & 4 \pi \omega \epsilon_{0} \mathcal{U}(\omega, T) \delta\left(\omega-\omega^{\prime}\right) \\
& \times \operatorname{Im}\left[\epsilon_{\alpha \beta}(\omega)\right] \delta\left(\mathbf{r}-\mathbf{r}^{\prime}\right) .
\end{aligned}
$$

Using these expressions we determine the ensemble average of the Poynting vector $\langle\mathbf{P}\rangle=\langle\mathbf{E} \times \mathbf{H}\rangle$ (for any dispersive and dissipative anisotropic medium) which reads (Einstein summation convention)

$$
\begin{aligned}
\left\langle P_{\gamma}\right\rangle= & \zeta_{\alpha \beta \gamma} 2 \operatorname{Re} \int_{0}^{\infty} \frac{d \omega}{2 \pi} \frac{2 \omega^{3} \mu_{0}}{c^{2}} \mathcal{U}(\omega, T) \\
& \times \int_{V} d \mathbf{r}^{\prime \prime}\left(\mathbb{G}^{\mathrm{EE}}\left(\mathbf{r}, \mathbf{r}^{\prime \prime}\right) \operatorname{Im}(\mathbf{\epsilon}) \mathbb{G}^{\mathrm{HE} \dagger}\left(\mathbf{r}, \mathbf{r}^{\prime \prime}\right)\right)_{\alpha \beta} .
\end{aligned}
$$

Here we have introduced the Levi-Civita tensor $\zeta_{\alpha \beta \gamma}$ and ${ }^{\dagger}$ denoting the conjugate transpose. In order to calculate the heat flux $\Phi^{o / e} \equiv\left\langle P_{z}\right\rangle$, we assume that the cavity is infinitely large so that we can replace it by a uniaxial half space. Inserting again the Green's dyadic [18,19] and integrating over this half space with volume $V$ we find after a lengthy calculation (see the Supplemental Material [8]) in the lossless limit, the relatively simple expression

$$
\Phi^{o / e}=\int_{0}^{\infty} \frac{d \omega}{2 \pi} \mathcal{U}(\omega, T) \int_{0}^{\infty} \frac{d k_{\perp}}{2 \pi} k_{\perp} \frac{\operatorname{Re}\left(k_{\|}^{o / e}\right)^{2}}{\left(k_{\|}^{o / e}\right)^{2}}
$$

for the mean Poynting vector or heat flux along the surface normal $\quad\left(k_{\|}^{o 2} \equiv\left(\omega^{2} / c^{2}\right) \epsilon_{\perp}-k_{\perp}^{2}\right.$ and $k_{\|}^{e 2} \equiv\left(\omega^{2} / c^{2}\right) \epsilon_{\perp}-$ $\left.k_{\perp}^{2}\left(\epsilon_{\perp} / \epsilon_{\|}\right)\right)$. Interestingly, the integral kernel $\left[\operatorname{Re}\left(k_{\|}^{o / e}\right)^{2} /\right.$ $\left.\left(k_{\|}^{o / e}\right)^{2}\right]$ can be interpreted as a transmission coefficient
$[29,30]$, which is one for all propagating modes $\left(k_{\|}^{o / e}\right.$ is purely real) inside the uniaxial medium and zero $\left(k_{\|}^{o / e}\right.$ is purely imaginary) otherwise. We have checked that the same result can be obtained by calculating the energy flow by using the DOS together with the group velocity so that also this result can be obtained without using the FDT. Hence, the maximum heat flux expressed by this equation is obtained when each mode contributes with a maximum transmission coefficient of one. Note, that our result is quite different from the results obtained in Refs. [31,32].

Evaluating this expression for the mean Poynting vector for the dielectric uniaxial material, first, we have

$$
\Phi_{D}^{o / e}=\int_{0}^{\infty} d \omega \mathcal{U}(\omega, T) \frac{\omega^{2}}{\pi^{2} c^{3}} \frac{c}{4} \frac{1}{2}\left\{\begin{array}{l}
\epsilon_{\perp} \\
\epsilon_{\|}
\end{array}\right\} .
$$

Note that up to this point the derived expressions for the mean Poynting vector are valid for dispersive materials. For nondispersive materials this simplifies to

$$
\Phi_{D}^{o / e}=\frac{c}{4} U_{\mathrm{BB}}^{s / p}\left\{\begin{array}{c}
\epsilon_{\perp} \\
\epsilon_{\|}
\end{array}\right\}=\Phi_{\mathrm{BB}}^{s / p}\left\{\begin{array}{c}
\epsilon_{\perp} \\
\epsilon_{\|}
\end{array}\right\} .
$$

When $\epsilon_{\perp}=\epsilon_{\|}=1$ we find again the usual BB result, i.e., the Stefan-Boltzmann law. On the other hand, inside a uniaxial material (as inside an isotropic material) with $\epsilon_{\perp}>1$ and $\epsilon_{\|}>1$ the radiative heat flux is larger than the $\mathrm{BB}$ value, which is a well-known fact [33].

In the case of HMs these results radically change. Before seeing this, let us first consider the OMs. For $\Phi_{\mathrm{I}}^{o}$ we find of course the same relation as for the dielectric anisotropic material, whereas as a consequence that there do not exist any OMs in a type II HM we find $\Phi_{\mathrm{II}}^{o}=0$. On the contrary, for the EMs we find

$$
\Phi_{\mathrm{I}}^{e}=\int_{0}^{\infty} d \omega \mathcal{U}(\omega, T) \frac{k_{\perp, \max }^{2}}{8 \pi^{2}} \quad \text { and } \quad \Phi_{\mathrm{II}}^{e}=\Phi_{\mathrm{I}}^{e}-\Phi_{D}^{e} .
$$

Hence, in the nondispersive case, where $k_{\perp, \max } \gg \frac{\omega}{c} \sqrt{\left|\epsilon_{\|}\right|}$ we have

$$
\Phi_{\mathrm{I}}^{e} \approx \Phi_{\mathrm{II}}^{e} \approx \frac{k_{\perp, \max }^{2}}{48 \hbar}\left(k_{B} T\right)^{2} .
$$

In this case, we see that the heat flux is proportional to $T^{2}$ and not anymore to $T^{4}$ as in the "classical" StefanBoltzmann law. Comparing this quantity with the classical $\mathrm{BB}$ results, we find $\Phi_{\mathrm{I} / \mathrm{II}}^{e} / \Phi_{\mathrm{BB}}^{p} \approx\left(k_{\perp, \max } l_{c}\right)^{2}\left(5 / 2 \pi^{2}\right)$. Hence, the normalized heat flux is proportional to $\left(k_{\perp, \max } l_{c}\right)^{2}$, which is due to the fact that the heat flux scales like the area of the projection of the isofrequency surface in $k$ space [34,35], or like the number of transversal modes $[29,30]$, respectively. This is quite astonishing, since 
for HMMs with a unit cell size $\Lambda$ of $10 \mathrm{~nm}$ and for natural HMs with a unit-cell size $\Lambda \approx 1 \AA$ we can now expect a hyperbolic BB heat flux 6 to 10 orders of magnitude larger than that of a usual BB at $T=300 \mathrm{~K}$. At cryogenic temperatures $l_{c}$ becomes very large so that this ratio can become even much larger. Note that the above limit is also the upper limit of heat radiation between two hyperbolic materials separated by a vacuum gap [36-40].

To summarize, we have extended the BB theory to arbitrary uniaxial materials. For dielectric anisotropic media we have seen that the thermodynamic properties of the photon gas inside such media are very similar to that of a classical BB. On the other hand, when these media are hyperbolic, the spectral energy distribution of radiation is shifted towards frequencies smaller than Wien's frequency. We have also shown that in contrast to the StefanBoltzmann law, the heat flux radiated by these media depends quadratically on their temperature. Nevertheless, the magnitude of heat flux carried by these media can be several orders of magnitude larger than the flux radiated by a classical BB and may even exceed the heat flux carried by conduction in superlattices [8]. Detailed derivations of the above relations and the underlying assumptions as well as more detailed discussions are given in the Supplemental Material [8].

S. L., A Y. P., and M. E. gratefully acknowledge financial support from the German Research Foundation (DFG) via SFB 986 " $\mathrm{M}^{3} "$, project $\mathrm{C} 1$.

*s.age.biehs@uni-oldenburg.de †pba@institutoptique.fr

[1] M. Planck, Ann. Phys. (Berlin) 309, 553 (1901).

[2] G. Kirchhoff, Monatsberichte der Akademie der Wissenschaften zu Berlin, sessions of Dec., 783 (1859).

[3] P. Yeh, Optical Waves in Layered Media (John Wiley \& Sons, New Jersey, 2005).

[4] D. R. Smith, W. J. Padilla, D. C. Vier, S. C. Nemat-Nasser, and S. Schultz, Phys. Rev. Lett. 84, 4184 (2000).

[5] D. R. Smith and D. Schurig, Phys. Rev. Lett. 90, 077405 (2003).

[6] L. Mandel and E. Wolf, Optical Coherence and Quantum Optics (Cambridge University Press, Cambridge, England, 2008).

[7] L. Novotny and B. Hecht, Nano-Optics (Cambridge University Press, Cambridge, England, 2012).

[8] See Supplemental Material at http://link.aps.org/ supplemental/10.1103/PhysRevLett.115.174301 for a detailed derivation of the DOS and heat flux as well as a discussion of a real hyperbolic metamaterial, which includes Refs. [9-15].

[9] W. Eckhardt, Z. Phys. B 46, 85 (1982).

[10] J. D. Jackson, Classical Electrodynamics (John Wiley \& Sons, Hoboken, 1999).
[11] M. Tschikin, S.-A. Biehs, P. Ben-Abdallah, S. Lang, A. Yu. Petrov, and M. Eich, J. Quant. Spectrosc. Radiat. Transfer 158, 17 (2015).

[12] E. W. Palik, Handbook of Optical Constants of Solids (Academic Press, San Diego, 1985).

[13] S. Strite and H. Morkoç, J. Vac. Sci. Technol. B 10, 1237 (1992).

[14] D. I. Florescu, V. M. Asnin, F. H. Pollak, R. J. Molnar, and C. E. C. Wood, J. Appl. Phys. 88, 3295 (2000).

[15] S.-M. Lee and D. G. Cahill, J. Appl. Phys. 81, 2590 (1997).

[16] G. S. Agarwal, Phys. Rev. A 11, 230 (1975).

[17] L.D. Landau and E. M. Lifshitz, Statistical Physics (Pergamon, Oxford, 1969).

[18] W. S. Weiglhofer, IEE Proceedings H 137, 5 (1990).

[19] A. S. Potemkin, A. N. Poddubny, P. A. Belov, and Y.S. Kivshar, Phys. Rev. A 86, 023848 (2012).

[20] W. Eckhardt, Opt. Commun. 27, 299 (1978).

[21] Z. Jacob, J.-Y. Kim, G. V. Naik, A. Boltasseva, E. E. Narimanov, and V. M. Shalaev, Appl. Phys. B 100, 215 (2010)

[22] I. I. Smolyaninov and E. E. Narimanov, Phys. Rev. Lett. 105, 067402 (2010).

[23] V. P. Drachev, V. A. Podolskiy, and A. V. Kildishev, Opt. Express 21, 15048 (2013).

[24] H. N. S. Krishnamoorthy, Z. Jacob, E. E. Narimanov, I. Kretzschmar, and V. M. Menon, Science 336, 205 (2012).

[25] D. W. Thompson, M. J. DeVries, T. E. Tiwald, and J. A. Woollam, Thin Solid Films 313-314, 341 (1998).

[26] M. Esslinger, R. Vogelgesang, N. Talebi, W. Khunsin, P. Gehring, S. de Zuani, B. Gompf, and K. Kern, ACS Photonics, 1, 1285 (2014).

[27] E. E. Narimanov and A. V. Kildishev, Nat. Photonics 9, 214 (2015).

[28] S. M. Rytov, Y. A. Kravtsov, and V. I. Tatarskii, Principles of Statistical Radiophysics 3 (Springer-Verlag, New York, 1989).

[29] S.-A. Biehs, E. Rousseau, and J.-J. Greffet, Phys. Rev. Lett. 105, 234301 (2010).

[30] P. Ben-Abdallah and K. Joulain, Phys. Rev. B 82, 121419 (R) (2010).

[31] E. E. Narimanov and I. I. Smolyaninov, arXiv:1109.5444.

[32] J. Liu and E. E. Narimanov, Phys. Rev. B 91, 041403(R) (2015).

[33] Z. Yu, N. Sergeant, T. Skauli, G. Zhang, H. Wang, and S. Fan, Nat. Commun. 4, 1730 (2013).

[34] M. Florescu, K. Busch, and J. P. Dowling, Phys. Rev. B 75, 201101(R) (2007).

[35] M. Florescu and K. Busch, J. Opt. A 11, 114005 (2009).

[36] I. S. Nefedov and C. R. Simovski, Phys. Rev. B 84, 195459 (2011).

[37] S.-A. Biehs, M. Tschikin, and P. Ben-Abdallah, Phys. Rev. Lett. 109, 104301 (2012).

[38] Y. Guo, C. L. Cortes, S. Molesky, and Z. Jacob, Appl. Phys. Lett. 101, 131106 (2012).

[39] S.-A. Biehs, M. Tschikin, R. Messina, and P. Ben-Abdallah, Appl. Phys. Lett. 102, 131106 (2013).

[40] S. I. Maslovski, C. R. Simovski, and S. A. Tretyakov, Phys. Rev. B 87, 155124 (2013). 